\title{
Metamodel-Based Multidisciplinary Design Optimization of a Deep-Sea Manganese Nodules Test Miner
}

\author{
Minuk Lee, ${ }^{1,2}$ Su-gil Cho, ${ }^{1}$ Jong-Su Choi, ${ }^{1,2}$ \\ Hyung-Woo Kim, ${ }^{2}$ Sup Hong, ${ }^{2}$ and Tae Hee Lee ${ }^{1}$ \\ ${ }^{1}$ Department of Automotive Engineering, Hanyang University, 222 Wangsimni-ro, Seongdong-gu, \\ Seoul 133-791, Republic of Korea \\ ${ }^{2}$ Ocean System Engineering Research Division, Korea Institute of Ocean Science \& Technology, \\ 32, 1312 Beon-gil, Yuseong-daero, Yuseong-gu, Daejeon-si 305-343, Republic of Korea
}

Correspondence should be addressed to Tae Hee Lee, thlee@hanyang.ac.kr

Received 21 February 2012; Accepted 8 May 2012

Academic Editor: Ioannis K. Chatjigeorgiou

Copyright (C) 2012 Minuk Lee et al. This is an open access article distributed under the Creative Commons Attribution License, which permits unrestricted use, distribution, and reproduction in any medium, provided the original work is properly cited.

A deep-sea manganese nodules test miner has not only coupled relationship between system components but also various design requirements of each system to meet the specified multitasks. To accomplish the multiobjectives of complex systems, multidisciplinary design optimization (MDO) is performed. Metamodels such as the kriging model and the response surface model are employed to reduce computational costs for MDO and to integrate component systems in a design framework. After verifying the accuracy of each metamodel, metamodel-based MDO for a deep-ocean test miner is formulated and performed. Finally, results and advantages of the proposed design methodology are discussed.

\section{Introduction}

Recently, a deep-sea mining system has received growing recognition for its development of plentiful marine mineral resources. It consists of a mining vehicle system, a transportation system, and a mother station. The deep-sea mining system is illustrated in Figure 1 [1]. In this research, we adopt a self-propelled mining vehicle system equipped with many devices. It collects mineral resources, especially manganese nodules, while traveling on cohesive soft soil of deep-sea floor about 5,000 meters depth. The transportation system conveys manganese nodules to the mother station through a flexible hose, a buffer, and riser pipes. The mother station stores the collected mineral resources and controls each subsystem with utility equipment. 


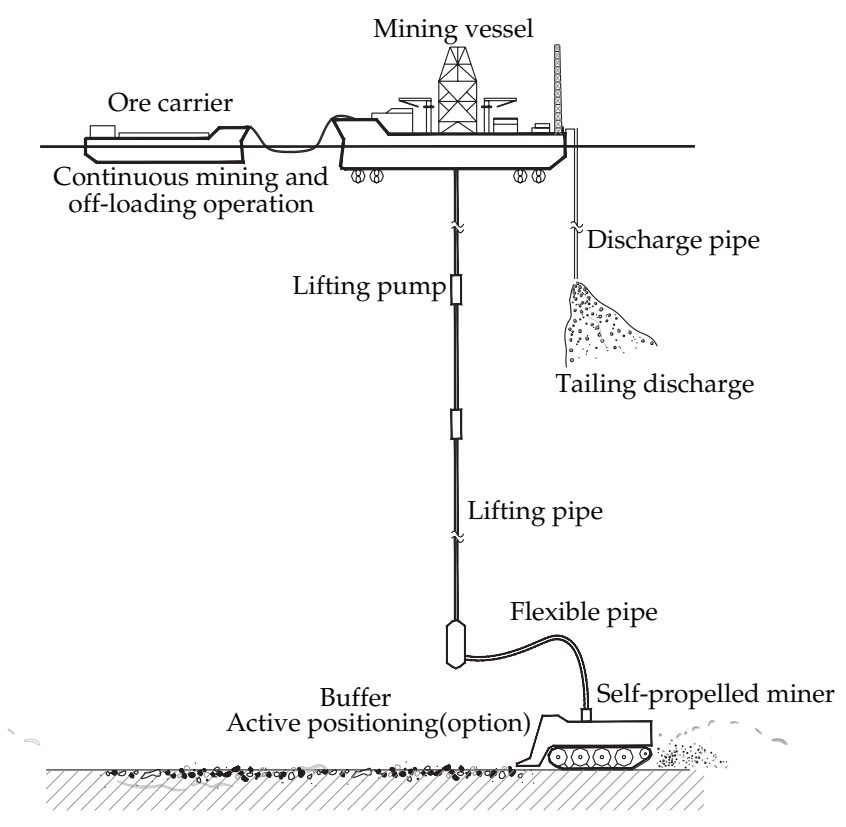

Figure 1: Schematic diagram of the integrated deep-sea mining system [1].

Among these systems, the mining vehicle system is a core component because it actually undertakes to pick up manganese nodules. It is verified by the axiomatic design method that the mining vehicle system must be designed first [2]. In this paper, we mainly consider the optimum design of a particular mining vehicle system - the test miner. The test miner is designed to verify the design concepts of the commercial deep-sea manganese nodules miner. Core components of the test miner are a crawler, a collector, and a chassis structure. There are a variety of design requirements related to mining vehicle systems. Crawlers should travel stably on the extremely soft soil of the deep sea while consuming the smallest amount of power if possible. The collector is required to efficiently pick up the manganese nodules, regardless of their sizes. The frame structure must support weight of mining vehicle system at launch and recovery as well as deep-sea external loads. Moreover, there are physical interactions between each part of the vehicle system. For example, collection rate of collector depends largely on the traveling performance of the crawler. Vehicle velocity is identified as a primary common design variable in both the collector and the crawler. The overall size of the collector and the crawler are directly related to the width and the length of the frame structure.

Hence the deep-sea mining vehicle system cannot be designed according to the objective of a single part only. Therefore, an optimization technique that can systematically control the conflicts of design objectives related to the mining vehicle system should be employed. These design requirements naturally lead to the concept of multidisciplinary design optimization (MDO). MDO is an optimization technique that can provide a synthetic optimum solution of a coupled system while satisfying complicated design constraints [3]. Thus MDO can reflect diverse design requirements of a number of discipline-coupled mining vehicle systems. For MDO of the test miner, responses that represent each design requirement should be computationally acquired and integrated into a design framework. 
To evaluate the motion of the mining vehicle, a computer dynamic simulation of the crawler moving on cohesive soil was employed [4] because it is very expensive to investigate the traveling performance by means of real-size prototype. Besides, one dynamic analysis of the crawler is quite time consuming because of its numerical complexity and nonlinearity. The considerable computational cost of dynamic analysis can be a burden for MDO because many dynamic analyses are required during the optimization process. Therefore, it is necessary to reduce the number of actual analyses. For the collector mounted on the crawler, we conduct a physical experiment to investigate the collection rate under the gathering condition as close to the actual environment as possible. However, it is not easy to conduct such a physical experiment for every optimization process too.

We use the mathematical approximation model, the so-called metamodel, such as the kriging model [5] or the response surface model [6] to replace and integrate expensive experiments or simulations in MDO. A metamodel can provide a mathematical relationship between design variables and responses by means of several simulations or experiments. To obtain a proper metamodel, we investigate suitable sampling techniques and accurate verification methodologies. Static and modal analysis for the frame structure responses was accomplished in order to ensure high strength and stiffness. Structural analysis is directly integrated with an optimizer because it takes only a few seconds.

In this paper, a brief concept of metamodel is introduced. Description of our mining vehicle system and design requirements is followed. Then, we formulate a metamodel-based MDO problem that minimizes power consumption while satisfying various design requirements; for instance, collection rate and traveling velocity are greater than specific values, respectively, while maximum stress on the frame should be less than the allowable stress. Based on MDO methodology, an optimum solution is obtained. Through such optimum results, we can obtain the fundamental design information for the deep-ocean mining vehicle system, and advantages of the proposed design methodology are discussed.

\section{Metamodel}

Most engineering design problems require simulations and/or experiments to evaluate design objectives and design constraints. For many problems, however, a single simulation can take many minutes, hours, or even days to complete. As a result, routine tasks such as design optimization become impossible since they require thousands or even millions of simulation evaluations. One way of alleviating this burden is constructing approximation models, known as metamodel. The basic concept of metamodel technique is to provide an approximate response through a moderate number of pre-executed simulations or experiments. To acquire accurate metamodel within a moderate number of simulations or experiments, adequate sampling strategies and validation techniques are applied in this study. The metamodel provides a designer with approximate design information for a complicated and expensive system through computationally cheap evaluations. That is, the metamodel can be employed as a simulator in the optimization process. Many alternative models and methods exist [7], but here we reviewed two most prevalent metamodels in the literature: the response surface model and the kriging model.

\subsection{Response Surface Model}

The response surface model (RSM) is a method for constructing global approximations to the system behavior based on results evaluated at various points in the design space. It is well 
known that RSM is a suitable approximation model for a physical experiment with random errors or relatively linear response. RSM is evaluated by assuming that the relationship between $y(\mathbf{x})$ and $\mathbf{x}$ is expressed as a polynomial with unknown coefficients that will be determined from preobserved data. For example, the 2nd-order polynomial RSM is of form as follows:

$$
\widehat{y}=\beta_{0}+\sum_{i=1}^{k} \beta_{i} x_{i}+\sum_{i=1}^{k} \sum_{j=1, i \leq j}^{k} \beta_{i j} x_{i} x_{j}
$$

where $\hat{y}$ represents the prediction of system response $y$. The parameters $\beta_{i}$ and $\beta_{i j}$ are determined by the least squares regression analysis, that is, best fitting the response to the specified data. In this paper, 2nd-order RSM is built from the physical experimental data of the responses to the collector.

\subsection{Validation of Response Surface Model}

For the regression model such as the RSM, analysis of variance (ANOVA) has been commonly used as a validation technique. In the ANOVA, the determination coefficient $r^{2}$ is frequently used to measure the fidelity of RSM [8] as follows:

$$
r^{2}=1-\frac{\mathrm{SSE}}{\mathrm{SSTO}}=1-\frac{\sum_{i=1}^{n}\left(Y_{i}-\widehat{Y}_{i}\right)^{2}}{\sum_{i=1}^{n}\left(Y_{i}-\bar{Y}\right)^{2}}
$$

where $n$ is the number of sample points. Total sum of square (SSTO) stands for the squared sum of total variations that are defined in terms of the deviations of the observations $Y_{i}$ around their mean $\bar{Y}$. Similarly, error sum of squares (SSE) represents the squared sum of the difference between observations $Y_{i}$ and the fitted value $\widehat{Y}_{i}$ at sampled points. We can interpret $r^{2}$ as how much variation metamodel can account for in total variation. Theoretically, when all observations fall on the fitted metamodel, then SSE $=0$ and $r^{2}=1$. At this time, the metamodel can account for all variation in the observations. By contrast, when the metamodel cannot capture the variation of observations at all, $\widehat{Y}_{i}$ approaches $\bar{Y}, \mathrm{SSE} \approx \mathrm{SSTO}$ and $r^{2} \approx 0$. It means that the effect of metamodel is negligible in explaining the variation of observations.

\subsection{Kriging Model}

Kriging originally comes from the field of geostatistics as a method to predict geological data, such as the thickness of ore layers [9]. Sacks and his coworkers exploited the kriging model as a prediction tool for engineering designs, where it is named as "Design and Analysis of 
Computer Experiments (DACE)" [5]. The kriging model is an interpolation model that is appropriate for deterministic responses of DACE. Recently, kriging model has been widely employed in the fields of a variety of engineering designs due to its excellent prediction performance and the useful statistical quantities [10]. The kriging model is formulated by the summation of global and local models as follows:

$$
y(\mathbf{x})=\mathbf{f}(\mathbf{x})^{\mathrm{T}} \boldsymbol{\beta}+\mathbf{Z}(\mathbf{x}),
$$

where $\mathbf{f}(\mathbf{x})^{\mathrm{T}} \boldsymbol{\beta}$ and $\mathbf{Z}(\mathbf{x})$ denote a global model and a localized model, respectively. The global model, $\mathbf{f}(\mathbf{x})^{\mathrm{T}} \boldsymbol{\beta}$, consists of the vector of polynomials and corresponding coefficients as follows:

$$
\begin{aligned}
& \mathbf{f}(\mathbf{x})=\left[f_{1}(\mathbf{x}), f_{2}(\mathbf{x}), \ldots, f_{p}(\mathbf{x})\right]^{\mathrm{T}} \\
& \boldsymbol{\beta}=\left[\boldsymbol{\beta}_{1}, \boldsymbol{\beta}_{2}, \ldots, \boldsymbol{\beta}_{p}\right]^{\mathrm{T}}
\end{aligned}
$$

where $p$ is the order of polynomial. Generally, the global model means a regression model, that is, mean model, $E[\mathbf{y}(\mathbf{x})]=\mathbf{f}(\mathbf{x})^{\mathrm{T}} \boldsymbol{\beta}$ throughout whole-design space. The localized model, $\mathbf{Z}(\mathbf{x})$, enables kriging model to capture nonlinear behavior of the response function. The systematic departure, $\mathbf{Z}(\mathbf{x})$, is assumed to be a Gaussian process with zero mean, that is, $E[\mathbf{Z}(\mathbf{x})]=0$ and covariance, $\operatorname{cov}\left[Z\left(\mathbf{x}_{i}\right), Z\left(\mathbf{x}_{j}\right)\right]$, for arbitrary two points $\mathbf{x}_{i}$ and $\mathbf{x}_{j}$. In kriging metamodel, it is important to note that the systematic departures at arbitrary two points are not independent of each other. Due to this feature, kriging model is noticeably distinguished from response surface model with identically independent residuals. Moreover, it is assumed that the covariance can be expressed in terms of the product of a process variance $\sigma^{2}$ and the correlation matrix $\mathbf{R}$ as follows:

$$
\operatorname{Cov}\left(Z\left(\mathbf{x}_{i}\right), Z\left(\mathbf{x}_{j}\right)\right)=\sigma^{2} \mathbf{R}\left(\mathbf{x}_{i}, \mathbf{x}_{j}, \boldsymbol{\theta}\right),
$$

where the process variance $\sigma^{2}$ is a scalar parameter that can be tuned to the responses by stochastic process, $\mathbf{R}$ is the correlation matrix that is related with the distance of each design points, and $\boldsymbol{\theta}$ is a vector of unknown correlation parameters to control the range of influence of nearby points that will be determined by maximum likelihood estimation.

Given these sampled outputs of the computer model, consider a linear estimator of the output

$$
\widehat{\mathbf{y}}(\mathbf{x})=\mathbf{c}^{\mathrm{T}}(\mathbf{x}) \mathbf{y}
$$


at any point $\mathbf{x} \in \Omega$. The kriging approach treats $\widehat{\mathbf{y}}(\mathbf{x})$ as a random function and finds the best linear unbiased predictor, $\mathbf{c}^{\mathrm{T}}(\mathbf{x}) \mathbf{y}$, which minimizes the mean-squared error of the prediction while satisfying the unbiased constraint. Consider the following:

$$
\begin{array}{cl}
\text { Find } & \mathbf{c}(\mathbf{x}) \\
\text { To minimize } & \operatorname{MSE}[\widehat{\mathbf{y}}(\mathbf{x})-\mathbf{y}(\mathbf{x})]=E\left[\left(\mathbf{c}^{\mathrm{T}}(\mathbf{x}) \mathbf{y}-\mathbf{y}(\mathbf{x})\right)^{2}\right] \\
\text { Subject to } & \mathrm{E}\left[\mathbf{c}^{\mathrm{T}}(\mathbf{x}) \mathbf{y}-\mathbf{y}(\mathbf{x})\right]=0 .
\end{array}
$$

Let the solution of the minimization problem of (2.7) be $\widehat{\mathbf{c}}(\mathbf{x})$. Finally, kriging predictor can be written as

$$
\widehat{\mathbf{y}}(\mathbf{x})=\widehat{\mathbf{c}}^{\mathrm{T}}(\mathbf{x}) \mathbf{y}
$$

In summary, the best linear unbiased predictor implies the linear predictor that minimizes the mean-squared error subjected to unbiased condition. In this study, kriging models are built from dynamic simulation data for responses of the crawler.

\subsection{Validation of Kriging Metamodel}

For interpolation model such as kriging metamodel, the validation scheme must be fundamentally different from ANOVA. It is due to the fact that interpolating model goes exactly through the observations; thereby, $r^{2}$ always becomes 1 . For this reason, the most prevalent method to examine the accuracy of interpolation model is cross-validation approach [11]. The basic idea of the approach is to leave out one or several sample data and to fit metamodels alternatively based on the rest of sample data. Next step is to evaluate the predicted values of metamodels on those leave-out data. Cross-validation error is defined in terms of the predicted values of leave-out metamodel and true response values. For example, leave-one-out cross-validation error is defined as

$$
C V=\sqrt{\frac{1}{n} \sum_{i=1}^{n}\left(\widehat{Y}_{-i}\left(\mathbf{x}_{i}\right)-Y\left(\mathbf{x}_{i}\right)\right)^{2}}
$$

where $Y\left(\mathbf{x}_{i}\right)$ is the true response value at $i$ th sample point $\mathbf{x}_{i}$, and $\widehat{Y}_{-i}\left(\mathbf{x}_{i}\right)$ denotes the predicted value at $\mathbf{x}_{i}$ using the metamodel created from the sample set which only $i$ th sample data is left out. Cross-validation technique is required too many combinations of metamodel to build up. Thus, it is difficult to conduct cross-validation approach for large problems.

To overcome above drawback, ANOVA using the additional validation sample set is introduced in this paper. Sequential maximum distance sampling technique is used to acquire additional validation point set that maximizes minimum distance from the previous sampling points. This sampling technique allocates sample points that one far from the existing 
sample points sequentially in the design space. If $r^{2}$ is less than target accuracy, kriging metamodel is rebuilt with added validation sample data set. And then, ANOVA is conducted with another validation sample data set. These iterative processes are terminated when $r^{2}$ reached the specified target value.

\section{Deep-Sea Manganese Nodules Test Miner}

The deep-sea mining vehicle system is an integrated system that includes the collector, the crawler, and the chassis structure. The test miner is required to travel stably on the semiliquid layer of deep seabed and simultaneously to collect manganese nodules as many as possible. Its traveling performance has a direct influence on the collection rate of manganese nodules. From a commercial viewpoint of deep-ocean mining system, low energy consumption of total system is one of the essential design requirements. In addition, low impact to suboceanic environment should be significantly taken into considerations due to the international convention for the detection of ocean ecological environment. Safety of frame structures should be considered as a design requirement because mining vehicle system is operated under about 500 bars and sustains the weight of all equipment. Moreover, there are a number of couplings among subsystems. For example, velocity is an input variable for the dynamic analysis of the crawler and simultaneously is primary design variable of the collector. Thus, we should consider these couplings for the design optimization of deep-ocean mining vehicle system.

In this research, we employed the concept design from the previous researches and determined the design variables considering features of the described design problem. We do not include couplings of the transportation system and mother station because the research of these systems is under process. Now, we describe design requirements of the test miner's components such as collector, crawler, and frame structures.

\subsection{Collector}

Collector is mounted on the front of self-propelled mining vehicle system and collects mineral resources from the ocean floor. The conceptual design of pick-up device should consider the economical, technical, and environmental aspects. Mechanical, hydraulic, and hybridcollecting methods have been proposed so far. Mechanical collecting type uses only mechanical system such as groom, shovel, and fork to pick up manganese nodule. This collecting mechanism has high pick-up efficiency. But this method has poor power efficiency and brings a bad influence on deep-sea ecosystem. Hydraulic collecting type is based on the Coanda nozzle effect; an object is apt to attach to a nearby surface when a free jet emerges from a nozzle. This collection method has simple structure, but there is a possibility of internal clogging up the ducts. Hybrid-collecting method, otherwise, can lift manganese nodules by a water jet and then move toward the conveyor with scrappers. It is fundamentally compromising method of above two types.

In this research, we adopt hybrid type that considers both reasonable collection rate and environment friendly feature [12]. Figure 2 represents the layout of the collector developed in our research. To collect manganese nodules reliably under irregular ground condition, collector must control the influx of water jet while maintaining a certain distance 


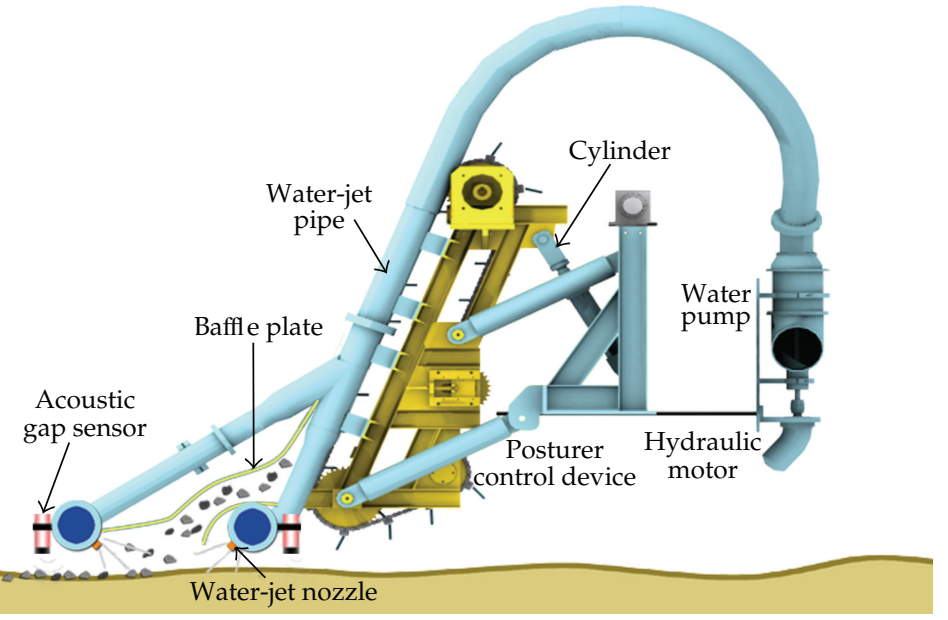

Figure 2: Configuration of hybrid type collector of the deep-ocean test miner.

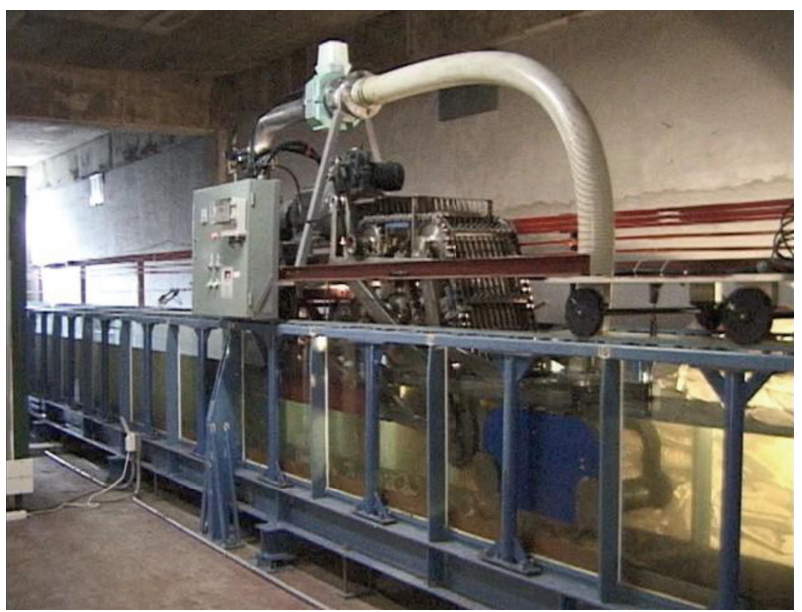

Figure 3: Test bed of hybrid type collector in 2-D flume.

from ground. Traveling velocity of crawler also should be controlled for efficient collection of man-ganese nodules without losing them.

The collecting performance is tested in 2-D flume that is similar to actual deep-seabed environment [13]. The collector performance test is illustrated in Figure 3. In this experiment, the collection rate of manganese nodules $\left(R_{\text {collector }}\right)$ was investigated. The collection rate is defined as the ratio of the number of collected nodules to the total number of nodules covered on bathtub before collecting them. Interval between nozzle and ground $(H)$, inflow of water jet per unit time $(Q)$, and drag velocity $\left(V_{g}\right)$ are considered for design variables 
for this experiment. Mass of collected manganese nodules per unit time can be computed as follows:

$$
\dot{M}=R_{\text {collector }} \times c \times b_{w} \times V_{g},
$$

where $c$ and $b_{w}$ denote the mass of manganese nodules covered on the unit area and collection width, respectively. In this study, we use metamodel for collector because it is hard to directly integrate the responses of physical experiment into MDO. As review in Section 2.1, RSM is a suitable approximation model for noisy physical experiment. Therefore, RSM is built from several physical experimental data tested in 2-D flume. The sampling for RSM is conducted as full factorial sampling that is often used for the design of physical experiments. Three levels for $H$, four levels for $Q$, and the three-level for $V_{g}$ are chosen, totally $36(3 \times 4 \times 3=36)$ experiments are conducted. For the collection rate, $r$-square coefficient of our RSM is 0.84 . In other words, this model has an 84-percent confidence compared with physical experiments. In physical experiments, the RSM is acceptable in general use when $r$ square coefficient is over the 0.8 , so this metamodel for collector is a reasonable approximate model.

From the view point of operation mining vehicle system, minimization of the power consumption is essential. The power consumption of collector can be calculated as follows:

$$
P_{\text {collector }}=\frac{\rho_{0} \times Q \times b_{w} \times h_{l}}{\operatorname{eff}_{p}}
$$

where $\rho_{0}, h_{l}$, and $\operatorname{eff}_{p}$ denote the specific weight of water, the head loss, and the efficiency of water jet pump, respectively.

\subsection{Crawler}

The deep-sea manganese nodules test miner has to run stably on cohesive soft seabed while simultaneously collecting the manganese nodules. Thus, crawler is adopted as the traveling equipment. However, it is not still easy for crawler to show the good mobility on extremely soft deep-sea soil. Deep-sea soil has so small shear strength that ill-traveling condition causes crawler to sink into seabed easily [14]. To develop a crawler with high performance, dynamic behavior should be investigated significantly under various traveling condition. To do this, ground and crawler models are carefully considered. Traffic ability of crawler on cohesive soft soil depends strongly on the proper driving resistance. The driving resistance is directly related to shear stress of deep-sea soil. Cohesive soil of deep sea is modeled by means of three relationships: pressure with respect to sinkage, shear stress with respect to shear displacement, and shear displacement with respect to the dynamic sinkage.

Rigid-body model of crawler is used to execute dynamic simulation as fast as possible. This rigid-body model is compared with multibody dynamic model against a variety of dynamic responses [15]. As a result, the rigid-body model shows quite-similar performance compared to multibody model. To evaluate mobility and power consumption of traveling vehicle, 

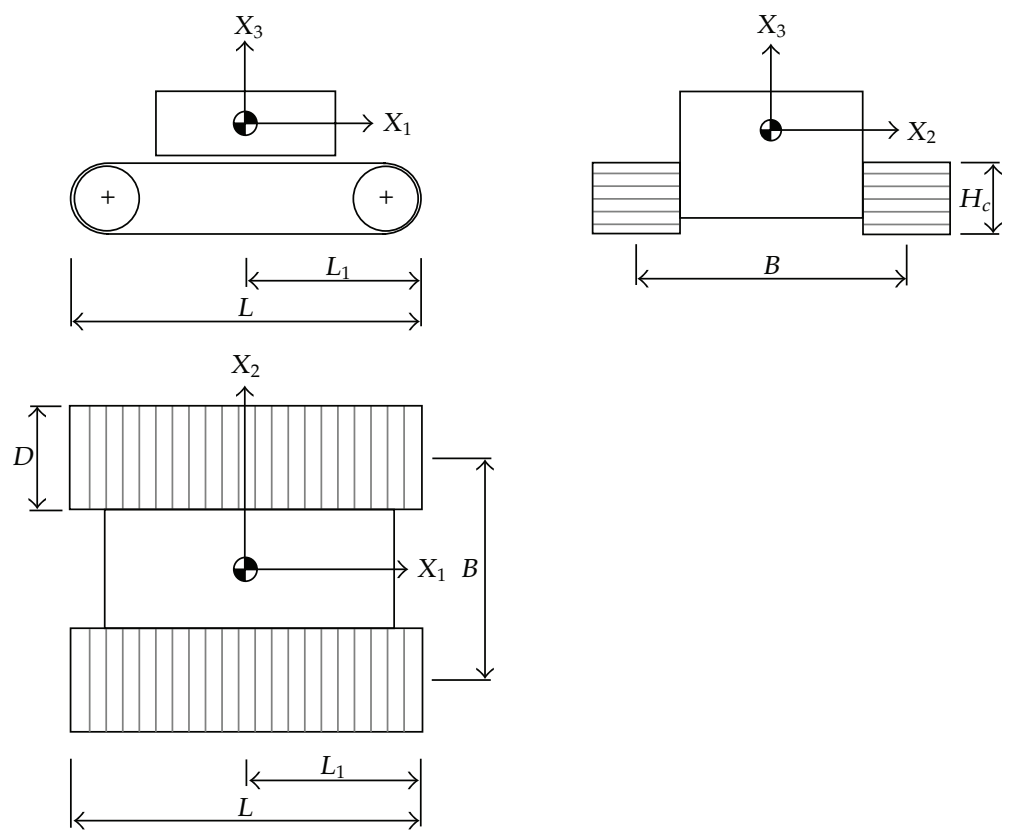

Figure 4: Configuration of tracked vehicle model and description of design variables.

next four responses: pitch angle $\left(\theta_{\text {pitch }}\right)$, vertical sinkage $\left(\delta_{z}\right)$, slip rate of track (slip) and torque of track (torq) are considered. Most of the manganese nodules collecting process are conducted at steady state of straight driving stage. Hence, mean values of steady state were adopted as representing responses of dynamic simulation. Slip rate is defined by ratio of actual forward-traveling velocity of crawler to driving velocity of track. And pitch angle is inclination of crawler toward the front. Usually, positive pitch angle (i.e., vehicle leans to the front) is preferred for compensation of rear sinkage of the vehicle due to slip. Power consumption of the crawler is related with torque of track. It can be calculated as follows:

$$
P_{\text {crawler }}=\frac{\text { torq }}{r_{\text {wheel }}} \times V_{t}
$$

where $V_{t}$ is track velocity, and $r_{\text {wheel }}$ is radius of track wheel. There are seven variables defined for the crawler: length of tracked vehicle $(L)$, track span $(B)$, track width $(D)$, total vehicle mass $(W)$, height of centroid $\left(H_{c}\right)$, distance from front to centroid of tracked vehicle $\left(L_{1}\right)$, and track velocity $\left(V_{t}\right)$. Configuration of the crawler is illustrated in Figure 4 . Note that drag velocity, $V_{g}$, is related to slip and $V_{t}$, that is, $V_{g}=(1-$ slip $) \times V_{t}$. That is, drag velocity is an output response of crawler including slip effect of cohesive soil.

Dynamic simulation of the tracked vehicle system is quite time consuming. Therefore, the considerable computational cost of dynamic analysis at one time can be a serious obstacle to conduct MDO. It is verified by comparative study that the kriging model is suitable to predict crawler dynamic responses [16]. In this study, the kriging metamodeling technique is introduced to approximate computationally expensive and highly nonlinear response of our dynamic simulation model. 


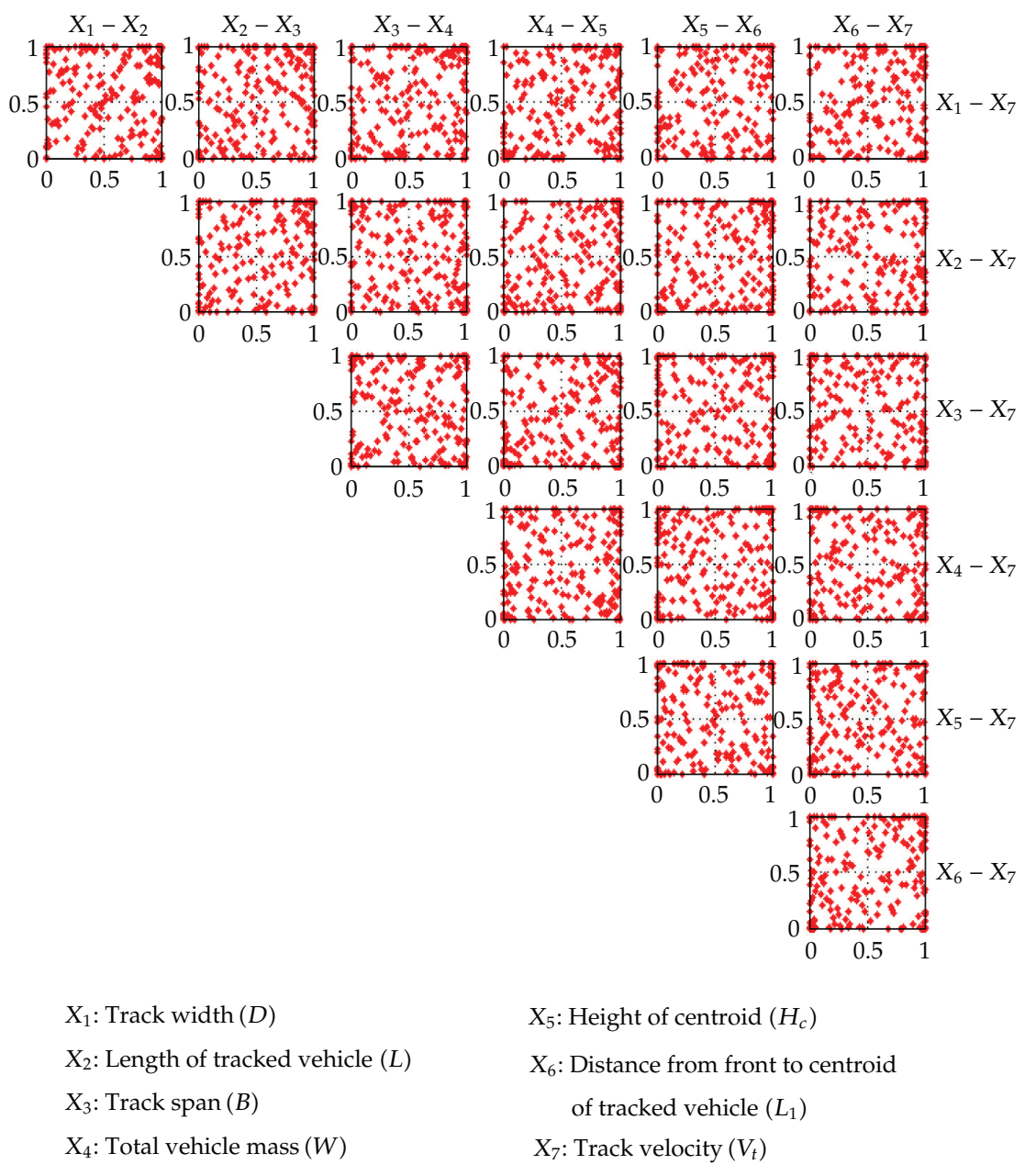

Figure 5: Projection plots of 180 sample points in normalized space for crawler kriging model.

To obtain an efficient and accurate metamodel, we must decide the adequate number of analyses and the distribution of design points. There are many sampling strategies based on this concept, such as design of experiment (DOE) and space-filling sampling. It is important to use the adequate number of sample points in order to obtain a metamodel with high fidelity. We select an initial sample set of 108 sampling points based on the optimal Latin hypercube sampling. Optimal Latin hypercube sampling is a compromise between optimal criterion such as entropy and Latin hypercube with the good projection properties. Thus, optimal Latin hypercube sampling finds the optimal design among all possible Latin hypercube sampling [17]. As a result, optimal Latin hypercube sampling can overcome poor space-filling properties of Latin hypercube samplings. Moreover, optimal Latin hypercube sampling is usually more efficient than other optimal criterion-based sampling strategies since it evaluates the criterion for candidates of Latin hypercube sampling.

Validation sample set with 36 sampling points that based on the maximum distance sampling technique is added sequentially until $r^{2}$ of ANOVA reached user-defined accuracy. Sequential maximum distance sampling adds points that maximize minimum distance from 

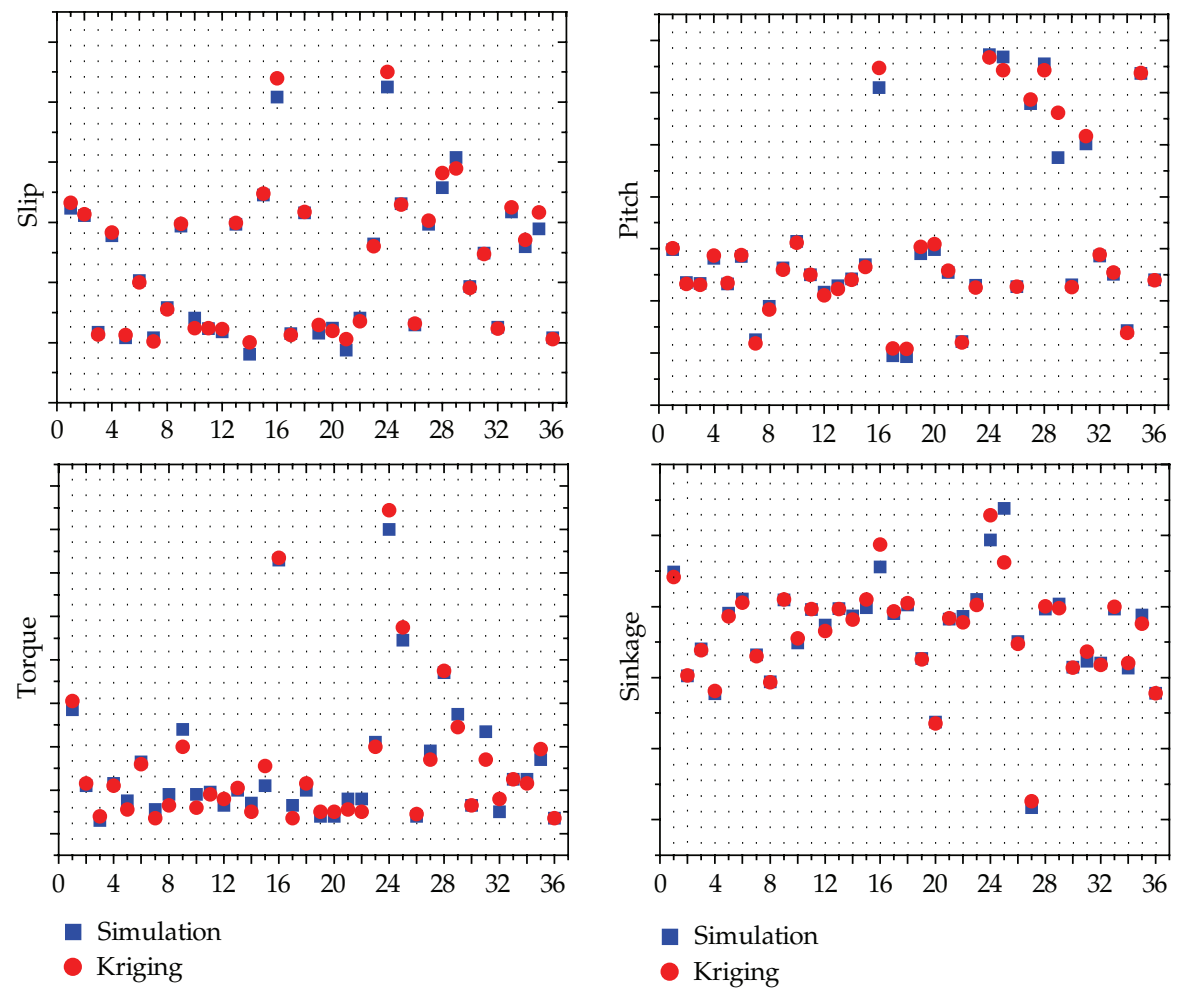

Figure 6: Comparison between crawler kriging model and simulation model for vertical sinkage, pitch angle, slip rate, and torque.

the previous sampling points for each sampling stage. This sampling technique allocates sample points sequentially at the emptiest location of the design space filled with the existing sample points. In this study, 180 sampling points, 108 points for Optimal Latin hypercube sampling technique and 72 points for sequential maximum distance sampling technique, are used to construct kriging metamodel. Figure 5 gives projection plots of 180 sampling points in the normalized space, which are well distributed over the entire design domain. The number of sample points is 5 times as many as the saturated points (the least number of simulations required for fitting a quadratic polynomial regression model with $k$ design variables, i.e., $m=(k+1)(k+2) / 2)$. In fact, 180 simulation points are not particularly excessive when compared to the 2-level full factorial design of 7 design variables $\left(2^{7}=128\right)$.

To validate the kriging model, the additional 36 sample points are obtained from sequential maximum distance approach. It is helpful to verify the accuracy of a metamodel through as small as possible an additional sample set since validation points are enough away from initial sample points. As shown in Figure 6, the kriging model can accurately predict the nonlinear dynamic responses of a tracked vehicle. To quantify the accuracy of each metamodel, Table 1 gives the $R$-square calculated at validation points.

\subsection{Chassis Structure}

Chassis structure supporting vehicle system must be strong and stiff enough to maintain the shape in pressures of approximately 500 bars of the deep sea and endure any handling 
Table 1: $R$ square of crawler kriging model.

\begin{tabular}{lcccc}
\hline & Sinkage & Pitch & Slip & Torque \\
\hline$R$ square & 0.9584 & 0.9895 & 0.9794 & 0.9907 \\
\hline
\end{tabular}

procedures, such as launch and recovery of the miner. For the chassis structure, structural analysis model was developed to evaluate the natural frequencies and structural strength by means of a commercial finite-element program. Structural analysis is directly integrated into MDO because it takes only a few second. Design variables for chassis structure are crosssectional area of main frame $\left(A_{m}\right)$, loading frame $\left(A_{l}\right)$, and collector position control frame $\left(A_{c}\right)$. Figure 7 shows the configuration of chassis frame and design variables. And responses of structure analysis are the maximum stress $\left(\sigma_{\max }\right)$ and the first natural frequency (freq).

\section{Formulation of MDO Problem for the Test Miner}

In this paper, we introduce MDO methodology that compromises contradictions of requirements in a complex system. To perform MDO, formulation of optimization problem should be carried out. The objective function of MDO problem is to minimize total power consumption $\left(P_{\text {total }}\right)$. Power consumption for operating the test miner is a significant factor to determine overall feature of deep-sea mining system. Especially, capacity of a power generator in mother station and a size of an umbilical cable are directly related with power consumption. These are significant for both the performance of the mining system and the commercial feasibility. Cost of the mother station and power generator increases exponentially for their capacity. Volume of umbilical cable can affect the mobility of the mining system. Thus, total power consumption $\left(P_{\text {total }}\right)$ adopted objective function of MDO problem.

The test miner should be traveling on the cohesive seabed reliably while pick up manganese nodules at least required amount of mineral resources. Required amount of the collected mineral resources is determined by commercial feasibility study [18, 19]. The posture of the vehicle has to be controlled within stable range to run on extremely cohesive soft soil.

Chassis structure supporting vehicle system must endure deep-sea pressure and handling forces such as launch and recovery. If these conditions are satisfied, the test miner can be operated reliably. But there are many couplings and contradictions for requirements between each subsystem. Foregoing requirements are considered for design constraints in MDO problem.

Coupling between subsystems must be investigated to formulate MDO problem. Since collector picks up manganese nodules by water jet flow, the driving velocity can affect both collector and crawler considerably. Vehicle-sizing parameters such as $L, B$, and $D$ are common design variable of collector and crawler. Coupled variable: $W, H_{c}$, and $L_{1}$ are calculated from chassis structure model to crawler. Dragged velocity is coupled with slip rate and track velocity. These interactions are illustrated in Figure 8.

MDO methodology requires integration of disciplines and many function calls. As reviewed in Sections 2 and 3, to reduce the number of actual simulation and the effort on interfacing between disciplines, metamodeling techniques are employed for crawler and collector. To formulate MDO problem, the above-mentioned objective function, design constraints and couplings are considered. In this study, the formulation of MDO problem for the 


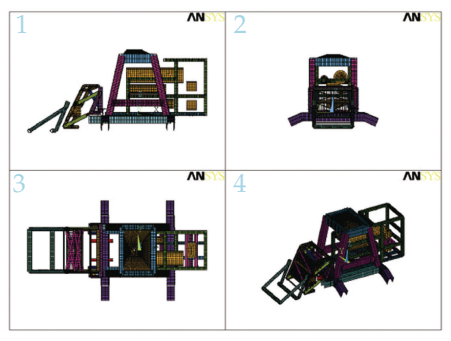

(a)

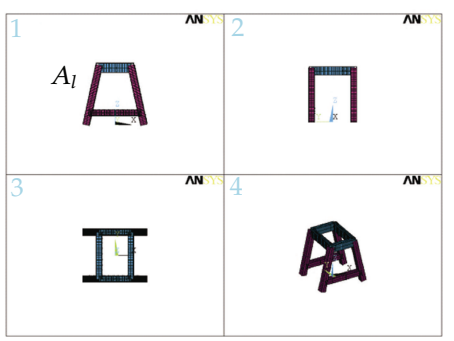

(c)

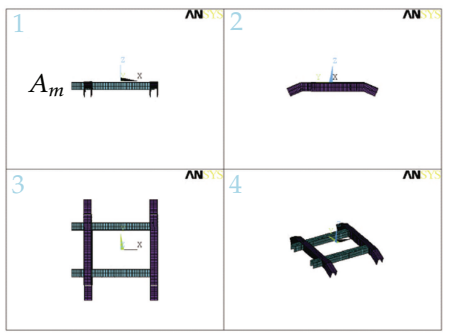

(b)

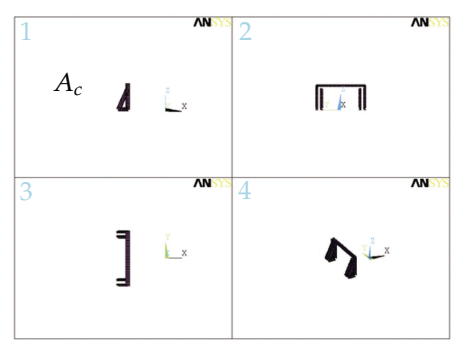

(d)

Figure 7: Configuration of chassis frame model and description of design variables.

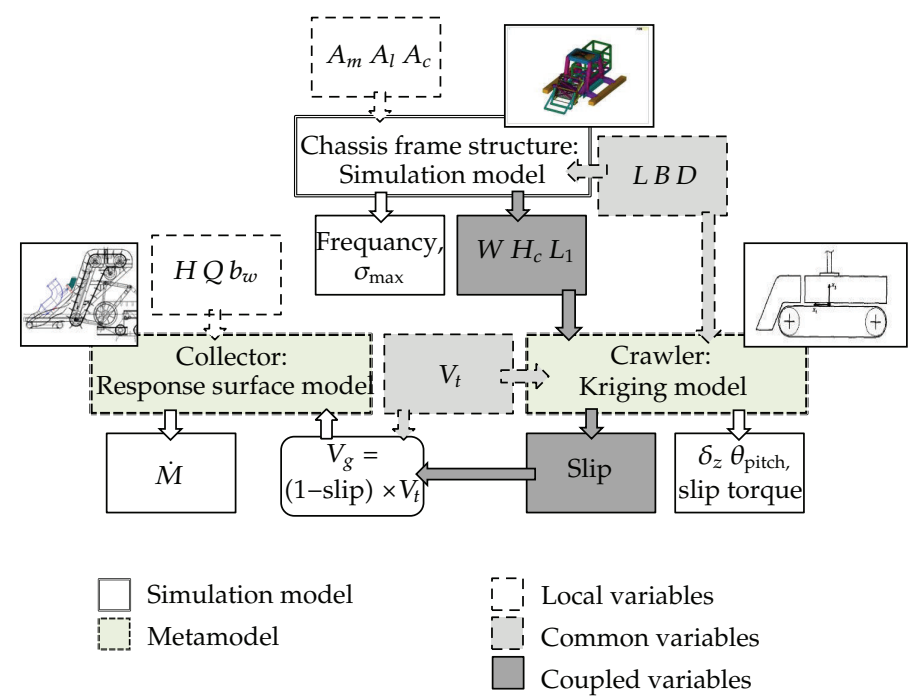

Figure 8: Coupled relations and variables of each subsystem of the test miner.

test miner is defined as follows:

Find $\quad D, L, B, A_{m}, A_{l}, A_{c}, V_{t}, b_{w}, H, Q$

To minimize $\quad P_{\text {total }}=P_{\text {collector }}+P_{\text {crawler }}$ 


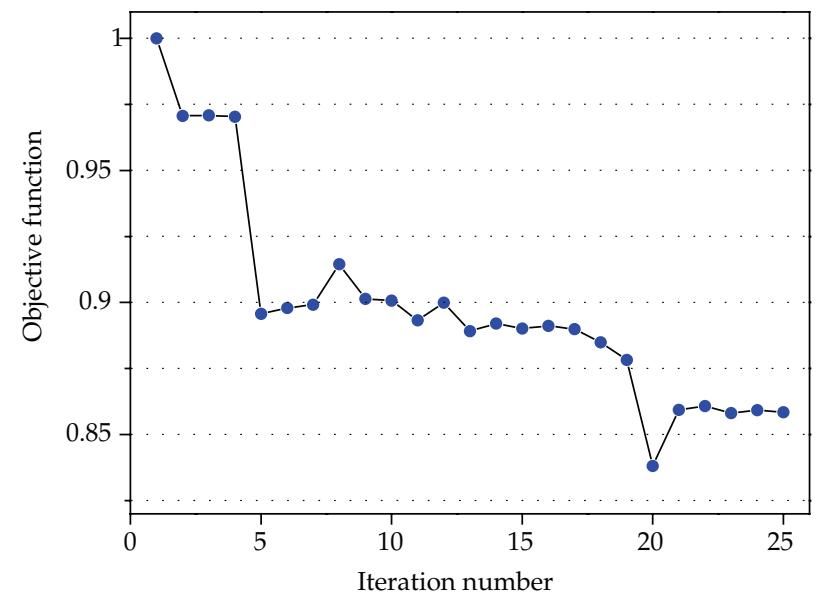

Figure 9: History of objective function during optimization process.

$$
\begin{array}{ll}
\text { Subject to } & \sigma_{\max }-\text { allowed stress } \leq 0 \\
& \text { allowed frequency }- \text { freq } \leq 0 \\
& \delta_{z}-\text { limit of vertical sinkage } \leq 0 \\
& \theta_{\text {pitch }}-\text { limit of pitch } \leq 0 \\
& \text { slip }- \text { limit of slip ratio } \leq 0 \\
& V_{g}-\text { limit of drag velocity } \leq 0 \\
& \text { mass of collected manganese nodules per unit time }-\dot{M} \leq 0 \\
& b_{w}-B-D \leq 0 .
\end{array}
$$

\section{Results and Discussions}

In this paper, optimization is performed by means of the commercial program, MATLAB. Sequential quadratic programming (SQP) is adopted as an optimization algorithm. Figure 9 shows the change of objective function in optimization process. As shown in Figure 11, the initial design is infeasible. However, all constraints are satisfied when optimum is achieved. We can derive the MDO result that the objective function, that is, total power consumption, was decreased about $14.1 \%$ while satisfying all design constraints. Results of MDO are summarized in Table 2. Design variables are expressed relative to the initial design between lower and upper bound, and responses are normalized to each constraint value, respectively. These are illustrated in Figures 10 and 11.

\section{Conclusion}

Multidisciplinary design optimization of a deep-sea manganese nodules test miner is defined and performed with the aid of metamodels. The test miner is composed of a crawler, 


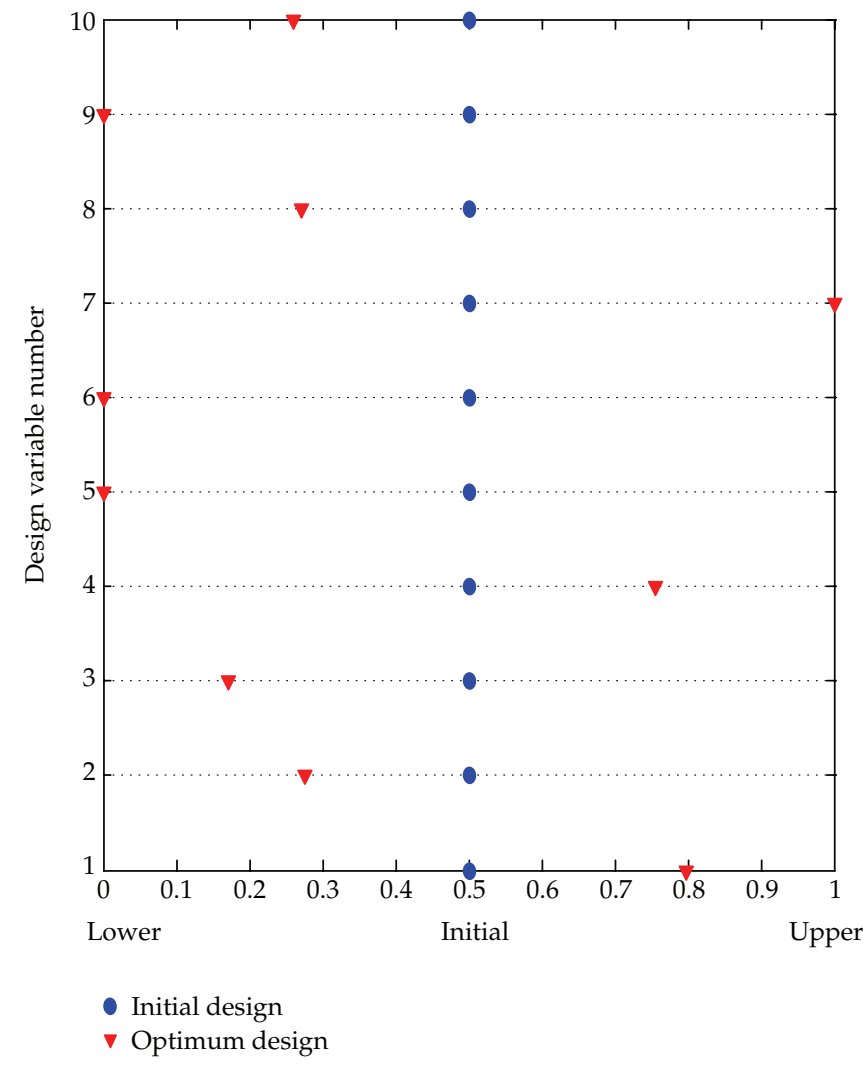

Figure 10: Changes of design variables at optimum with respect to the initial design value.

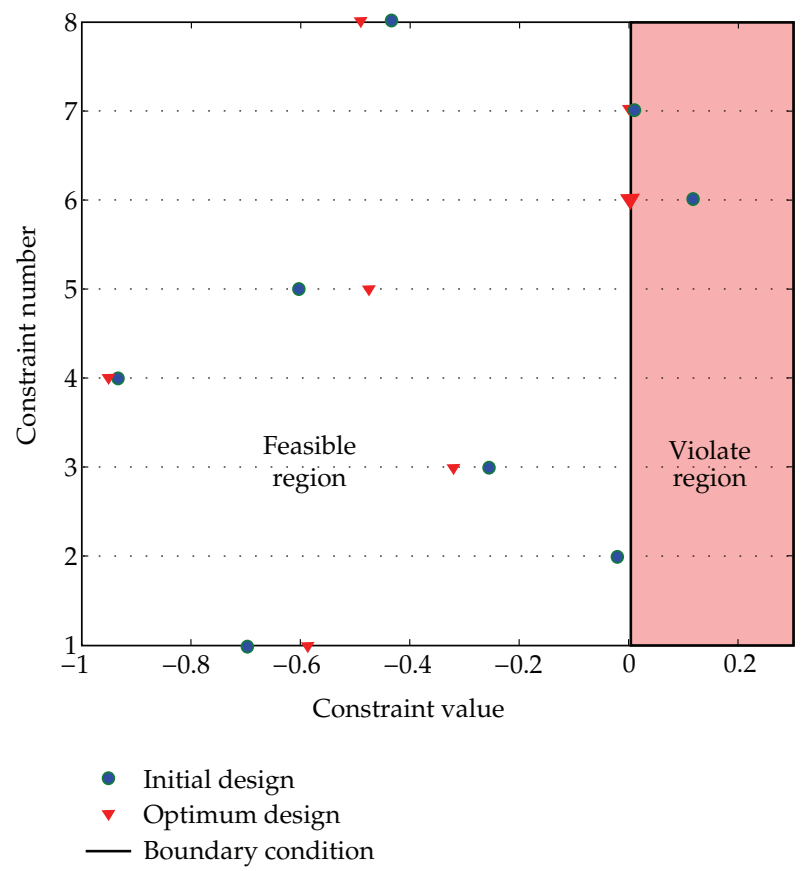

Figure 11: Changes of design constraints at optimum with respect to the initial design. 
Table 2: Result of MDO for the test miner.

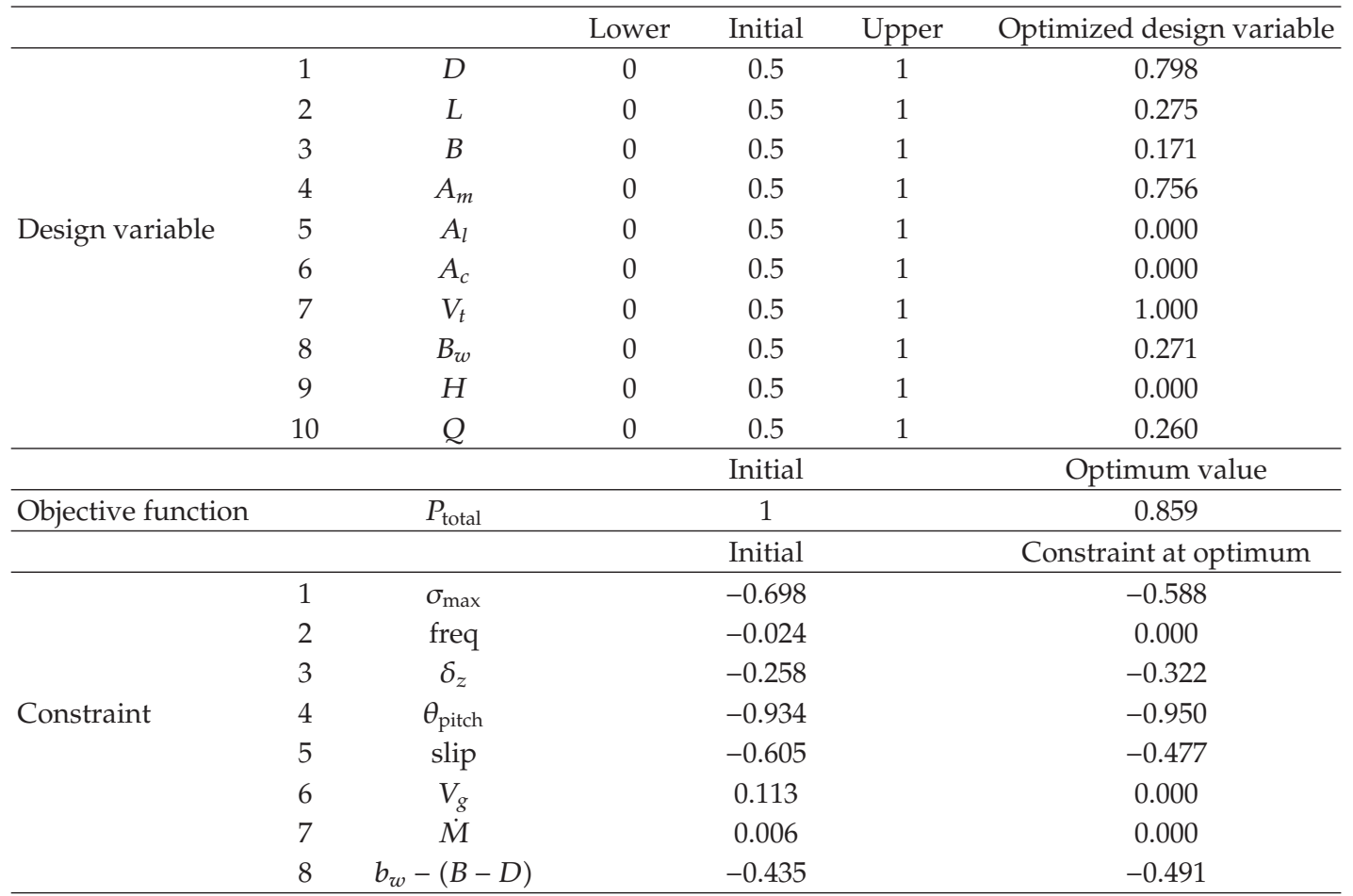

a collector, and a chassis structure. To build up metamodel for each performance of the subsystem, metamodel techniques such as response surface model and kriging metamodel are employed and appropriated sampling methods are chosen. Based on this research, we can drive the following conclusions.

(i) To formulate MDO problem, design requirements and couplings between each subsystem of the test miner are investigated.

(ii) MDO methodology requires an integration of many disciplines and a lot of function calls. To reduce the number of actual simulation and the effort on interfacing between disciplines, metamodeling techniques are employed for the crawler and the collector.

(iii) MDO is successfully performed using MATLAB with metamodels.

(iv) Metamodel-based MDO reduces the power consumption by nearly $14.1 \%$ compared to the initial design while satisfying specified all design constraints.

\section{Acknowledgments}

The contents of this paper are a part of the R\&D project, Technology Development of Deepseabed Mining System for Manganese Nodules, sponsored by the Ministry of Land and Transport and Maritime Affairs of Korea. The authors appreciate this Ministry's full support for this research. 


\section{References}

[1] S. Hong, H. W. Kim, J. S. Choi et al., "A self-propelled deep-seabed miner and lessons from shallow water tests," in Proceedings of the ASME 29th International Conference on Ocean, Offshore and Arctic Engineering (OMAE '10), June 2010.

[2] J. S. Choi, S. Hong, H. W. Kim, T. K. Yeu, and T. H. Lee, "Design evaluation of a Deepsea manganese nodule miner based on axiomatic design," in Proceedings of the 6th International Society of Offshore and Polar Engineers, Ocean Mining Symposium (ISOPE OMS '05), pp. 163-167, October 2005.

[3] R. J. Balling and J. Sobieszczanski-Sobieski, "Optimization of coupled systems: a critical overview of approaches," AIAA Journal, vol. 34, no. 1, pp. 6-17, 1996.

[4] S. Hong, H. W. Kim, and J. S. Choi, "Transient dynamic analysis of tracked vehicles on extremely soft cohesive soil," in Proceedings of the 5th ISOPE Pacific/Asia Offshore Mechanics Symposium, pp. 100-107, November 2002.

[5] J. Sacks, W. J. Welch, T. J. Mitchell, and H. P. Wynn, "Design and analysis of computer experiments," Statistical Science, vol. 4, pp. 409-435, 1989.

[6] R. H. Myers and D. C. Montgomery, Response Surface Methodology, John Wiley and Sons Inc, New York, NY, USA, 1995.

[7] T. W. Simpson, J. D. Peplinski, P. N. Koch, and J. K. Allen, "Metamodels for computer-based engineering design: survey and recommendations," Engineering with Computers, vol. 17, no. 2, pp. 129-150, 2001.

[8] J. Neter, M. H. Kutner, C. J. Nachtsheim, and W. Wasserman, Applied Linear Statistical Models, McGrawHill, 1996.

[9] G. Matheron, "Principles of geostatistics," Economic Geology, vol. 58, pp. 1246-1266, 1963.

[10] M. J. Sasena, Flexibility and efficiency enhancements for constrained global design optimization with Kriging approximations [Ph.D. thesis], University of Michigan, 2002.

[11] J. C. Helton and F. J. Davis, "Latin hypercube sampling and the propagation of uncertainty in analyses of complex systems," Reliability Engineering and System Safety, vol. 81, no. 1, pp. 23-69, 2003.

[12] K. S. Min, J. Y. Shim, S. Hong, and J. S. Choi, "Conceptual design of a hybrid pick-up device for deep ocean mining," in Proceedings of ISOPE (OMS '97), pp. 91-94, 1997.

[13] S. Hong, J. S. Choi, J. H. Kim, and C. K. Yang, "Experimental study on hydraulic performance of hybrid pick-up device of manganese nodule collector," in Proceedings of the 3rd ISOPE Ocean Mining Symposium, pp. 69-77, November 1999.

[14] J. S. Choi, S. Hong, and S. B. Choi, "Probability distribution for the shear strength of seafloor sediment in the KR5 area for the development of manganese nodule miner," Ocean Engineering, vol. 38, pp. 2033-2041, 2011.

[15] H. W. Kim, S. Hong, and J. S. Choi, "Comparative study on tracked vehicle dynamics on soft soil: single-body dynamics vs. multi-body dynamics," in Proceedings of the 5th ISOPE Ocean Mining Symposium, pp. 132-138, jpn, September 2003.

[16] T. H. Lee, J. J. Jung, S. Hong, H. W. Kim, and J. S. Choi, “Prediction for motion of tracked vehicle traveling on soft soil using kriging metamodel," International Journal of Offshore and Polar Engineering, vol. 17, no. 2, pp. 132-138, 2007.

[17] M. Meckesheimer, A. J. Booker, R. R. Barton, and T. W. Simpson, “Computationally inexpensive metamodel assessment strategies," AIAA Journal, vol. 40, no. 10, pp. 2053-2060, 2002.

[18] F. Søreide, T. Lund, and J. M. Markussen, “Deep ocean mining reconsidered a study of the manganese nodule deposits in Cook Island," in Proceedings of the 4th ISOPE Ocean Mining Symposium, pp. 88-93, September 2001.

[19] K. Nam, W. Oh, and S. Kwon, "An economic feasibility study of manganese nodule in Korea area: Clarion-Clipperton Fracture Zone," Ocean and Polar Research, vol. 26, no. 2, pp. 187-197, 2004. 


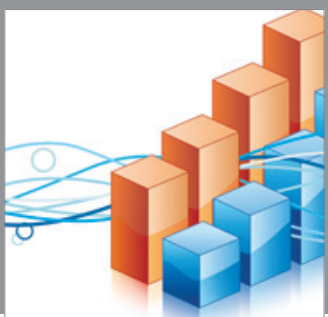

Advances in

Operations Research

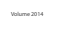

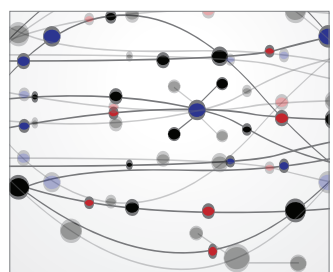

\section{The Scientific} World Journal
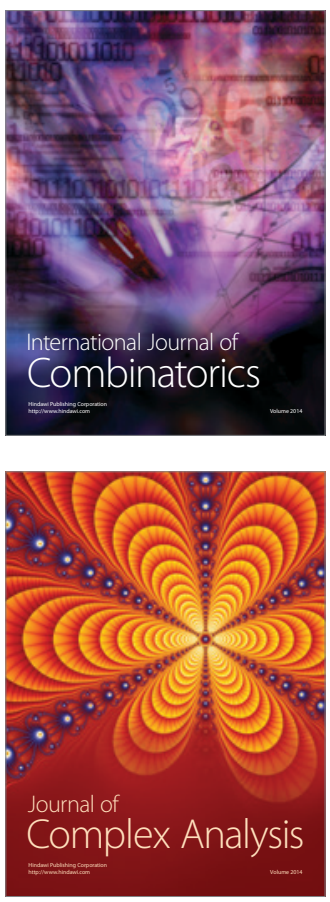

International Journal of

Mathematics and

Mathematical

Sciences
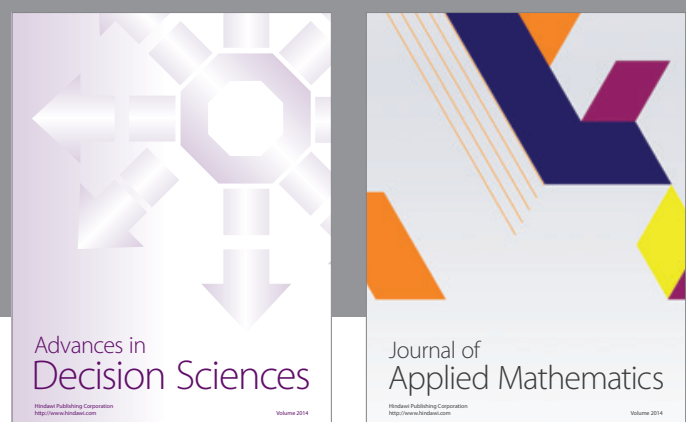

Journal of

Applied Mathematics
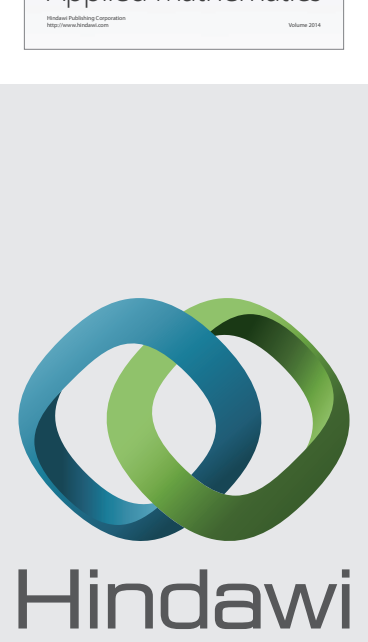

Submit your manuscripts at http://www.hindawi.com
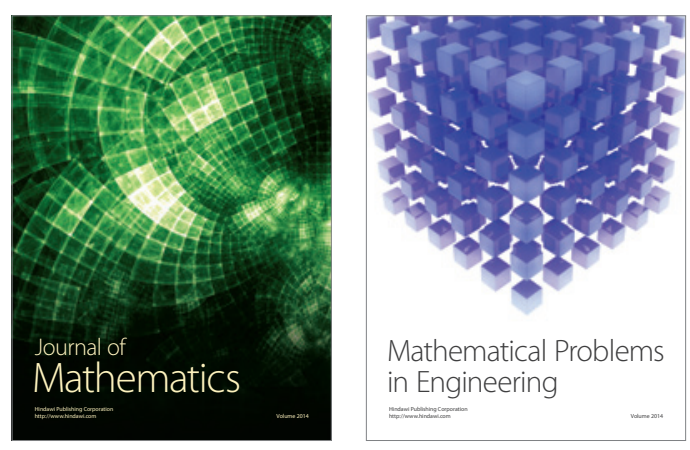

Mathematical Problems in Engineering
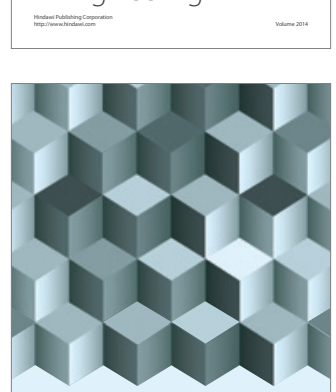

Journal of

Function Spaces
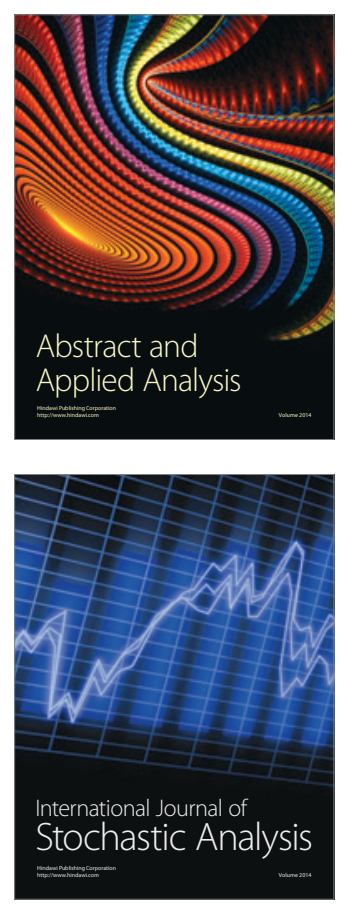

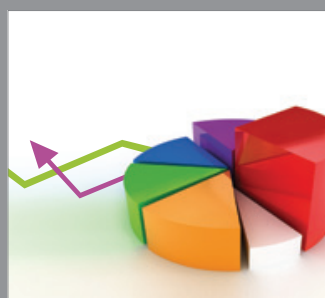

ournal of

Probability and Statistics

Promensencen
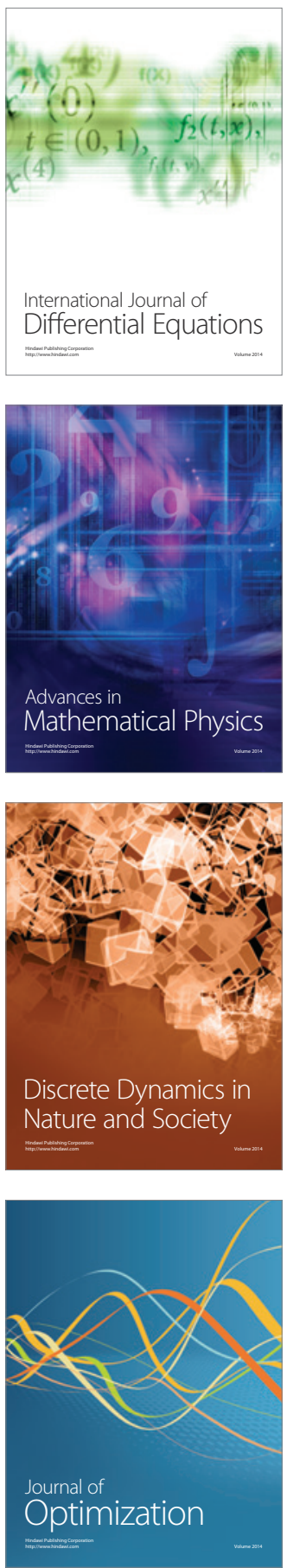